\title{
Identification of Safety Metrics for Airport Surface Operations
}

\author{
Wendy A. Okolo*, Molly O'Connor ${ }^{\dagger}$, Lilly Spirkovska ${ }^{\dagger}$ \\ NASA Ames Research Center, Moffett Field, CA 94035, USA \\ Herman Soyfer $\ddagger$ \\ San Jose State University, San Jose, CA 95192, USA
}

\begin{abstract}
A large fraction of safety incidents occurs on the ground during airport surface operations. Although these incidents are mostly non-fatal with a few exceptions, they are high profile incidents that remain a source of concern for the National Transportation Safety Board (NTSB), the Federal Aviation Administration (FAA), major airlines, and other stakeholders of the National Airspace System (NAS). These incidents have historically been mitigated by implementing changes to regulations, policies, and procedures over time. This approach has minimized but not eliminated the risk of occurrences. It is thus important to develop integrated techniques to assess, model, and prevent these incidents by analyzing the risk and likelihood of occurrence and communicating results of the analysis to decision-making personnel who can mitigate and prevent incidents in real time. The research presented in this report builds on prior work of researchers at the NASA Ames Research Center who developed an automated framework, Real-Time Safety Monitoring (RTSM), to enable monitoring and prediction of the safety of the NAS. In the RTSM framework, hazards to flight are translated to safety metrics such as wake vortex encounters or loss of separation, that can be modeled and analyzed offline and also predicted and monitored in real time (online). The intent of this report is to integrate predictable incidents that occur during surface and ground operations into the safety portfolio of the RTSM project by (i) identifying suitable information sources from which ground incidents can be studied, (ii) developing safety metrics correlated with surface operations, and (iii) recommending suitable data sources that can be quantified and used for the computation of pertinent safety metrics.
\end{abstract}

\footnotetext{
*Intelligent Systems Division, Discovery and Systems Health Area, MS 269-3, AIAA Member

$\dagger$ Intelligent Systems Division, Discovery and Systems Health Area, MS 269-3

‡Electrical Engineering Department, Graduate Student
} 